Philosophie ANTIQUE

\section{Philosophie antique}

Problèmes, Renaissances, Usages

19 | 2019

L'épicurisme antique

\title{
David LEFEBVRE, Dynamis. Sens et genèse de la notion aristotélicienne de puissance
}

Paris, Librairie philosophique J. Vrin, 2018 (Bibliothèque d'histoire de la philosophie), 607 p., ISBN 978-2-7116-2765-3

\section{Fabienne Baghdassarian}

\section{CpenEdition}

\section{Journals}

Édition électronique

URL : https://journals.openedition.org/philosant/1875

DOI : 10.4000/philosant.1875

ISSN : 2648-2789

Éditeur

Éditions Vrin

\section{Édition imprimée}

Date de publication : 31 octobre 2019

Pagination : 186-188

ISBN : 978-2-7574-2534-3

ISSN : 1634-4561

Référence électronique

Fabienne Baghdassarian, « David lefEBvre, Dynamis. Sens et genèse de la notion aristotélicienne de puissance », Philosophie antique [En ligne], 19 | 2019, mis en ligne le 14 mars 2019, consulté le 02 décembre 2022. URL : http://journals.openedition.org/philosant/1875 ; DOI : https://doi.org/10.4000/ philosant.1875

\section{(c) $(1)$}

Creative Commons - Attribution - Pas d'Utilisation Commerciale - Pas de Modification 4.0 International - CC BY-NC-ND 4.0

https://creativecommons.org/licenses/by-nc-nd/4.0/ 
each line. At the same time, it offers numerous philological, linguistic and grammatical indications that make this study a section suitable for scholars. This is a good option as it allows concentrating in an independent space several details that could have hindered the reading of the first sections.

In this way, the book combines parts proper for a broader audience that may be legitimately interested in Aeschines and the Socratic philosophies, without depriving scholars of the detailed discussion that must be undertaken in order to promote this field. This point is especially important because this work belongs to a cutting-edge area and it is prerequisite for its growth that it becomes increasingly visible in contemporary historiography beyond the limits of the philological community.

It was a good asset to insert an appendix dedicated to the history of modern editions of Aeschines' extant texts, including those from the beginning of the eighteenth century and those that were published between 1850 and 1912. From this study, it is possible to appreciate the evolution in the area and how it fell into disrepair during the twentieth century until it recovered their significance in recent years. In this context, the work of Pentassuglio constitutes a substantial contribution to the development of this field, and it will surely gain a well-deserved place in the tradition of studies on the Socratic philosophies.

Claudia MÁrsico

Universidad de Buenos Aires - CONICET / ANCBA

David Lefebvre, Dynamis. Sens et genèse de la notion aristotélicienne de puissance, Paris, Librairie philosophique J. Vrin, 2018 (Bibliothèque d'histoire de la philosophie), 607 p., ISBN 978-2-7116-2765-3.

On ne peut que saluer la parution de cet ouvrage important, dont le sous-titre exprime d'emblée l'originalité et la force : élucider le sens de la notion aristotélicienne de dynamis par l'étude de son histoire ou de sa genèse ( $c f$. p. 7 et 32). Cette méthode de travail répond à la volonté de débrouiller les fils d'une ambiguité bien connue de la notion aristotélicienne de puissance, qui se partage entre deux acceptions principales, explicitées en Metaph. $\Theta$ : d'une part, la puissance kata kinesin, qui désigne un principe actif ou passif de changement, par opposition à l'impuissance ; de l'autre, un sens plus ontologique, qui exprime un mode d'être par contraste avec l'energeia. Ce sont la nature et les raisons de cette « double orientation de la dynamis aristotélicienne » que l'ouvrage se propose d'éclairer. Mais, s'il s'agit là d'une question somme toute classique, dans le champ des études aristotéliciennes, l'ouvrage innove en se proposant de dépasser les deux méthodes ordinairement mises en œuvre pour résoudre cette ambiguité : tantôt par un commentaire interne du livre $\Theta$, tantôt par une lecture génétique cherchant à replacer ce double sens de la puissance au sein d'une évolution d'Aristote (lecture génétique dont l'auteur montre les limites méthodologiques et textuelles, p. 14-22). Car si l'ouvrage ne manque évidemment pas d'offrir une étude précise des thèses et de la construction du livre $\Theta$, s'il ne dénie pas non plus, à l'évidence, tout intérêt à une perspective historique, il formule en revanche l'hypothèse que l'ambiguité de la puissance aristotélicienne ne peut trouver sa solution qu'à la condition de sortir du seul cadre des textes d'Aristote, pour les replacer au sein d'une histoire plus large de la notion de dynamis, à la fois sémantique et conceptuelle, afin de comprendre ce que le Stagirite en recueille, accepte, modifie ou prolonge.

À l’originalité de cette méthode se joint celle du point de départ que David Lefebvre 
(D. L.) attribue à l'histoire grecque de la puissance. En montrant que les premières occurrences, chez Homère, de la dynamis ou des termes apparentés s'inscrivent toutes dans l'usage de la formule kata dynamin, exprimant, sous couvert de restriction, les moyens d'action dont un être dispose, il fait l'hypothèse que c'est dans cette idée de capacité propre que résident tout à la fois le sens initial de la notion de dynamis et le principe de son développement, bien plutôt que dans l'idée de force ou de pouvoir actif qu'on place d'ordinaire volontiers à l'origine de la notion ( $c f$. J. Souilhé, Étude sur le terme $\triangle \mathrm{YNAMI} \Sigma$ dans les dialogues de Platon, Paris, Félix Alcan, 1919).

L'ouvrage se divise en trois parties, retraçant les trois ou quatre principales étapes de l'histoire grecque de la dynamis jusqu'à Aristote. La première partie, intitulée « Puissance et limite » (p. 35-180) précise les sens initiaux de la dynamis, en revenant sur la clause kata dynamin, qui borne les premières occurrences de la dynamis chez les auteurs archaïques. D. L. montre que, d'Homère à Hésiode, Démocrite et Xénophon, s'approfondit un même sens de la dynamis, entendue non comme force, mais comme ensemble de moyens ou de capacités propres à un être et exprimant tout à la fois sa nature et ses limites - sa condition. Il poursuit en indiquant comment ce premier usage de la dynamis se prolonge jusque chez Platon et Aristote où, dans des contextes métaphysiques différents, il sert à exprimer l'adaptation toujours un peu déficiente du sensible à l'intelligible ou de la matière à la forme.

La deuxième partie se consacre à « La formation de la notion de puissance » chez Platon (p. 181-344). L'auteur montre, en effet, que c'est en réponse à une nouvelle acception de la dynamis comme toute-puissance que Platon, le premier, constitue la puissance en notion philosophique et en formule une définition qui, dans le prolongement du sens initial de la dynamis, en exprime les limites intrinsèques. En s'appuyant notamment sur le Gorgias, la République, le Sophiste ou l'Euthydème, D. L. retrace l'émergence, chez Platon, de deux horizons conceptuels et problématiques différents : d'un côté, Platon définit la dynamis comme une capacité monovalente de faire ou de subir quelque chose, déterminée par son domaine et son opération ; de l'autre, il l'ouvre à l'indétermination, en en subordonnant la valeur à l'usage qu'on en fait.

Capacité déterminée par un ergon et caractéristique de la nature d'un être, ou bien capacité indéterminée et ouverte à une multiplicité d'usages, telles seraient les deux sources platoniciennes de l'ambiguïté aristotélicienne de la puissance. C'est le développement qui y conduit que la troisième partie, intitulée «Puissance, capacité et force $\gg$ (p. 345-539), s'efforce de retracer. D. L. commence ainsi par montrer comment Aristote assume l'indétermination axiologique que la notion d'usage, progressivement remplacée par celle d'energeia, introduit au sein de la puissance (dans les Topiques, par exemple), et surtout, comment il étend cette indétermination au plan ontologique, à travers la notion de puissance des contraires, puis de la contradiction, élaborée en $\Theta$. Cette indétermination de la dynamis fait l'objet d'une critique de la part d'Aristote, à ceci près qu'il ne s'agit plus seulement de viser l'indétermination éthique de la toute-puissance, mais l'indétermination ontologique de ce qui peut être ou ne pas être et qui achève d'ôter à la dynamis la possibilité, pourtant élaborée chez Platon, de dire l'être. Quant à l'autre orientation de la puissance aristotélicienne, elle se rattacherait, via Platon, au sens initial de la puissance. L'auteur examine ainsi successivement la manière dont Aristote, en $\Theta$ essentiellement, définit cette puissance comme principe de changement, puis y inscrit des limites, de sorte que la dynamis, entendue comme capacité articulée à une fin, peut continuer, comme chez Platon, d'exprimer la nature d'un être et d'en permettre la définition. Dans un dernier chapitre, D. L. précise sous l'effet de quelles contraintes conceptuelles la notion de puissance croise parfois chez Aristote celle de force. L'analyse 
du mélange, à cet égard, est particulièrement éclairante, Aristote renouant, sur ce point, avec un sens ancien, mais second, de la dynamis, que l'auteur fait remonter à Parménide, Alcméon et à la médecine hippocratique, et définissant la puissance non pas seulement comme une capacité déterminée, mais comme un pouvoir en lutte avec d'autres et, dans cette mesure, comme une force intensive.

Pas plus qu'on ne peut, par ce résumé trop succinct, rendre justice à l'ampleur et à la subtilité des analyses proposées dans ce livre, on ne saurait en évoquer ici, même rapidement, l'ensemble des résultats. À l'évidence, il s'agit là de bien plus que d'un nouvel ouvrage sur la dynamis aristotélicienne. En plus d'offrir une lecture remarquable de la notion et de la terminologie de la dynamis depuis Homère, qui parvient à en réarticuler les différents sens philosophiques et extra-philosophiques (capacité, force, valeur, sens d'un mot, puissance mathématique), l'ouvrage se présente aussi comme une étude à part entière de la conception platonicienne de la dynamis. Telle n'en est sans doute pas la raison d'être initiale; il n'en demeure pas moins que D. L. fournit ici l'une des études les plus complètes et les plus éclairantes sur le sens et le traitement platoniciens d'une notion, à laquelle aucun Dialogue n'est explicitement consacré, mais dont il est clair, à la lecture de l'ouvrage, qu'elle fait l'objet d'une réflexion constante, technique et stratégique de la part de Platon.

Peut-être, du reste, est-ce dans cette attention soutenue au texte platonicien que se situe l'une des contributions les plus déterminantes de l'ouvrage à l'étude de la notion aristotélicienne de puissance. Ce n'est certes évidemment pas une idée neuve que de tâcher d'éclairer, sur ces questions, le travail conceptuel aristotélicien à partir de celui de Platon. C'est cette piste, entre autres exemples, que suit $S$. Menn, dans son article

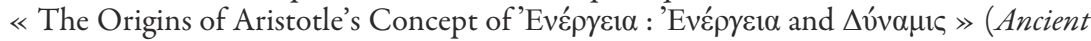
Philosophy 14, 1994, p. 73-114), afin de retracer l'émergence du couple acte-puissance chez Aristote, depuis la distinction platonicienne entre ktesis et chresis. C'est encore sur l'analyse de ce même lien fondamental entre Aristote et Platon que s'ouvre l'ouvrage de J. Beere (Doing and Being : an Interpretation of Aristotle's Metaphysics Theta, Oxford, Oxford University Press, 2009) qui voit dans la gigantomachie du Sophiste la matrice des problématiques aristotéliciennes concernant l'acte et la puissance. L'ouvrage de D. L. prolonge et déplace cette piste d'enquête en la faisant porter sur le concept de dynamis lui-même, et non plus seulement sur le couple qu'il forme avec l'energeia; il la systématise aussi en montrant nettement, plus nettement qu'à titre d'indications liminaires, combien le concept aristotélicien de dynamis hérite directement de l'ambiguïté que Platon y a introduite et qu'Aristote affronte directement, résolu à tirer toutes les conséquences d'une divergence entre deux approches que Platon semble avoir cherché à tenir ensemble.

Une autre des réussites de la méthode d'enquête adoptée dans l'ouvrage tient à sa capacité d'articuler, avec plus de souplesse qu'une étude interne ne l'aurait peutêtre permis, les différents sens de la dynamis aristotélicienne à l'échelle de la globalité du corpus. Par la perspective historique élargie qui est la sienne, l'ouvrage ne parvient pas seulement à éclairer ou à bien structurer les différents horizons conceptuels de la dynamis, il en montre aussi les sources et les logiques, à la fois internes et externes, et rend ainsi possible une compréhension de la notion plus souple, qui sait en marquer les différences, tout en rendant intelligibles les articulations et les croisements que le corpus donne constamment à voir. 